\title{
Digital analysis of the geometric variability of bamboo poles in bending
}

\author{
Rodolfo Lorenzo ${ }^{1, *}$, Leonel Mimendi ${ }^{1}$, and Haitao $\mathrm{Li}^{2}$ \\ ${ }^{1}$ Department of Civil, Environmental and Geomatic Engineering, University College London, London, UK \\ ${ }^{2}$ College of Civil Engineering, Nanjing Forestry University, Nanjing, 210037, China
}

\begin{abstract}
The importance of sustainable materials in the building industry has grown during the last decades due to the negative impact that industrialised materials have caused over the environment as a consequence of the rapid increase of urban areas, especially in developing countries. To alleviate this problem, research has focused on the utilisation of bamboo, a readyto-use natural material with a high potential of playing an important role in the future development of the building industry, however, the limitations of the material have not been properly addressed and therefore, the current use of bamboo in the building industry is negligible. This paper presents a digital approach to analyse the structural effects that the inherent geometric variability causes in bamboo poles subjected to bending effects. In contrast with current methodologies that apply the use of conventional tools to determine average geometric properties of a bamboo pole, this work adopted a combination of material testing and 3D scanning of a series of bamboo elements, in order to define suitable parameters for structural analysis that accurately represents the geometric variability of the poles. The results confirmed that the geometric variability produces significant effects that had not been considered before in the mechanical characterisation of bamboo, and that could not have been possible to identify without the use of digital technology. This work encourages the future development of methodologies that implement the use of digital technology to increase the structural understanding of bamboo and therefore, offer a robust platform for the general implementation of bamboo culms in the building industry.
\end{abstract}

\section{Introduction}

During the last century industrial products became the main material supply for the building sector, so that currently nearly 40 per cent of the global greenhouse gas emissions are due to construction activities [1]. The negative impact of the building industry on the environment is expected to rise during the following years, especially in developing countries where urbanisation rates are the highest [2]. For decades bamboo has been identified as one of the most promising alternatives to help reduce the environmental impact caused by the building sector, but its current use as a building material is negligible because the inherent geometric, mechanical and physical variabilities of individual culms reduce the reliability of bamboo as a structural element.

Bamboo culm resembles what is called a fast-growing natural composite material due to the natural arrangement of its microstructure [3] which is composed of unidirectional fibres (vascular bundles) strictly arranged parallel to the main axis of the pole, surrounded by a matrix (parenchyma) that constitutes the main body of the wall-thickness and holds the fibres in place [4]. More than a bond between fibres, the matrix is considered to carry compression forces, whereas the vascular bundle withstands the tension ones [5]. Bamboo geometry can be described as a long tapered hollow cylinder, with intermittent transverse membranes along its axis, known as "nodes". These geometric characteristics increase bamboo efficiency as structural element due to the high strength-to-weight ratio when compared to similar industrialised materials.

During the last 20 years, researchers have focused on the determination of bamboo geometric [6-8] and mechanical [9-11] properties with the aim of characterising the material and provide engineering support for the structural implementation of bamboo elements, however, the application of conventional methodologies for material characterisation have not been adequate for bamboo due to the complexity of its geometric and mechanical characteristics. On one hand, the geometric characteristics of bamboo include tapering; nodes randomly distributed along the height; section properties varying along the pole; and out-of-straightness [12]. In spite of the relative complexity in geometric 
features, the international standard for the determination of physical and mechanical characterisation of bamboo poles, ISO-22157-1 [13], considers that dimensions should be hand measured and averaged out for each individual pole, neglecting the effect of geometric variability.

On the other hand, researchers have found different ranges of mechanical properties when studying the same species, for example, Chung and $\mathrm{Yu}$ [7] found that for Mao Jue bamboo, the compression strength varied from 37 to $152 \mathrm{~N} / \mathrm{mm}^{2}$ whilst Lo et al [14] found that for the same specie the compression strength ranged from 46 to $65 \mathrm{~N} / \mathrm{mm}^{2}$. In addition to the variation between individual poles, there is also proof that the mechanical properties tend to vary from bottom to top, and from the inner to the outer layer across the wall thickness within the same pole [5]. In spite of the efforts made by researchers, there are yet no detailed guidelines on how to predict the mechanical behaviour efficiently and accurately, accounting for the material variability of individual poles. The conventional methodology of assigning characteristic values to a batch of bamboo culms [13], fails to describe the range of mechanical variation among bamboo and can potentially lead to an over-dimensioning of the elements due to overconservative safety factors to account for the material uncertainty.

With the emergence of new digital technologies and development of computer software, different areas of the industrial sector have taken advantage of novel nondestructive and reverse engineering methodologies. These methodologies have been applied as a replacement for the conventional acquisition of product data and the performance of physical tests through the implementation of digital workflows suitable for the characterisation of product properties. An example of a digital approach applied on bamboo culms is proposed in a research project [15] focused on developing a methodology to digitise and therefore, quantify the geometric variability of individual bamboo poles. The methodology was based on a $3 \mathrm{D}$ scanning process that acquires data points to then be merged into a polygon mesh [16] which becomes the digital representation of the bamboo poles. The method accurately captures the geometry and can allow the user to quantify geometric imperfections, such as variability in cross section properties, node distribution and out-ofstraightness.

This work focuses on the analysis of structural effects caused by the geometric variability, inherent in every pole, through finite element (FE) modelling of five bamboo poles based on the accurate digital information provided by $3 \mathrm{D}$ scan data. In addition, the possibility of determining the structural effects due to the mechanical variability of the material is discussed on the basis of a similar digital framework where both the geometric and mechanical characteristics of individual bamboo poles are accurately quantified.

\section{Experimental tests}

\subsection{Materials and methodology}

A benchmark for the general geometric and mechanical properties of the bamboo samples used in this work was determined following the standard ISO-22157-1 [13]. The samples consisted of five Moso bamboo poles (Phylostachys Pubescens), 3 to 4 years of age, harvested in Jiangsu Province in China. After felling, the bamboo was treated with a local procedure of carbonisation and oven-dried, before being transported to the Structures Laboratory at Nanjing Forestry University, where the bamboo was kept for three weeks until testing. On arrival, the average moisture content (MC) of the poles was $11.7 \%$, with no significant variation recorded at the time of testing. The physical properties for each bamboo pole were measured according to [13] and summarised in Table 1 .

Table 1. Summary of geometric and physical properties

\begin{tabular}{cccccc}
$\begin{array}{c}\text { Moso } \\
\text { Bamboo }\end{array}$ & $\begin{array}{c}\text { Diameter } \\
\mathrm{mm}\end{array}$ & $\begin{array}{c}\text { Thickness } \\
\mathrm{mm}\end{array}$ & $\begin{array}{c}\text { Length } \\
\mathrm{mm}\end{array}$ & $\begin{array}{c}\text { Density } \\
\mathrm{kg} / \mathrm{m}^{3}\end{array}$ & $\begin{array}{c}\mathrm{MC} \\
\%\end{array}$ \\
\hline R03 & 96 & 9.7 & 3000 & 723 & 11.6 \\
$\mathrm{R} 05$ & 89 & 7.8 & 3000 & 677 & 12.8 \\
$\mathrm{R} 08$ & 99 & 9.0 & 3000 & 773 & 9.4 \\
$\mathrm{R} 09$ & 89 & 9.0 & 3000 & 788 & 13.4 \\
R10 & 92 & 7.4 & 3000 & 724 & 11.2
\end{tabular}

Mechanical testing consisted of a 4-point bending test as described in [13], the general set-up of which is shown in Figure 1. The set-up consisted of a hydraulic servocontrolled actuator Popwil MAS-300 with a maximum capacity of $300 \mathrm{kN}$ and a stroke length of $500 \mathrm{~mm}$. The load was evenly distributed by a transfer-beam connected by an articulation to the actuator. The free span of the test was set to three meters and the point loads were evenly distributed at $1 / 3$ and $2 / 3$ of the span. The end of the bamboo pole closer to the root (bottom) was rested on a roller-like support, whereas the opposite end of the bamboo (top) rested on a pin-like support. Wooden saddles of approximately three diameters of length were positioned between the supports/loading points and the bamboo to allow an adequate load distribution. The load was applied until failure at a constant rate of $0.5 \mathrm{~mm} / \mathrm{s}$. Vertical displacement was measured at the mid-span using a displacement measurement laser sensor Sick ODPrecision with a maximum range of $400 \mathrm{~mm}$ and an accuracy of $0.4 \mathrm{~mm}$. The load and displacement data was gathered with a multi-channel data logger TML TDS-530 at a rate of 1 sample per second.

It is important to note that the natural geometric variability of bamboo will always lead to an unknown degree of deviation from the test set-up described in [13] as it does not take this inherent variation into account. As shown in Figure 1, the main deviation consisted in the position and alignment of the loads and supports. The standard suggests that wooden saddles should be used for the loads and supports, and that these saddles should be located over the nodes (see Figure 3 in [13]). This suggestion might be based on studies that show that the node offers radial support to the culm and prevent a failure for local buckling [6]. Not only the variable 
internodal length in bamboo poles make this requirement very difficult to comply with, but also a more recent study found that the nodes appear to be the region where the failure originates so that the contribution of the nodes as a reinforcing element is negligible [17]. For these reasons, the load/support points were simply located at the same position for all the samples, regardless of the location of the nodes, and the pair of saddles were replaced by a single, longer saddle (approximately three diameters in length) to spread the load and prevent local crushing.

Also, the alignment of support and loading points in a single vertical plane is not feasible due to the natural out- of-straightness of the poles. As shown in Figure 2, the supports were aligned with a vertical plane that passes through the axis of the transfer beam ( $X$ axis), however the loading points were, in all cases, out of the plane even when the bamboo was let to "find its own position" as required in [13]. In addition, due to the natural taper of the poles, the applied forces will not necessarily be perpendicular to the axis of the bamboo generating a component of these forces in the longitudinal direction along the pole.
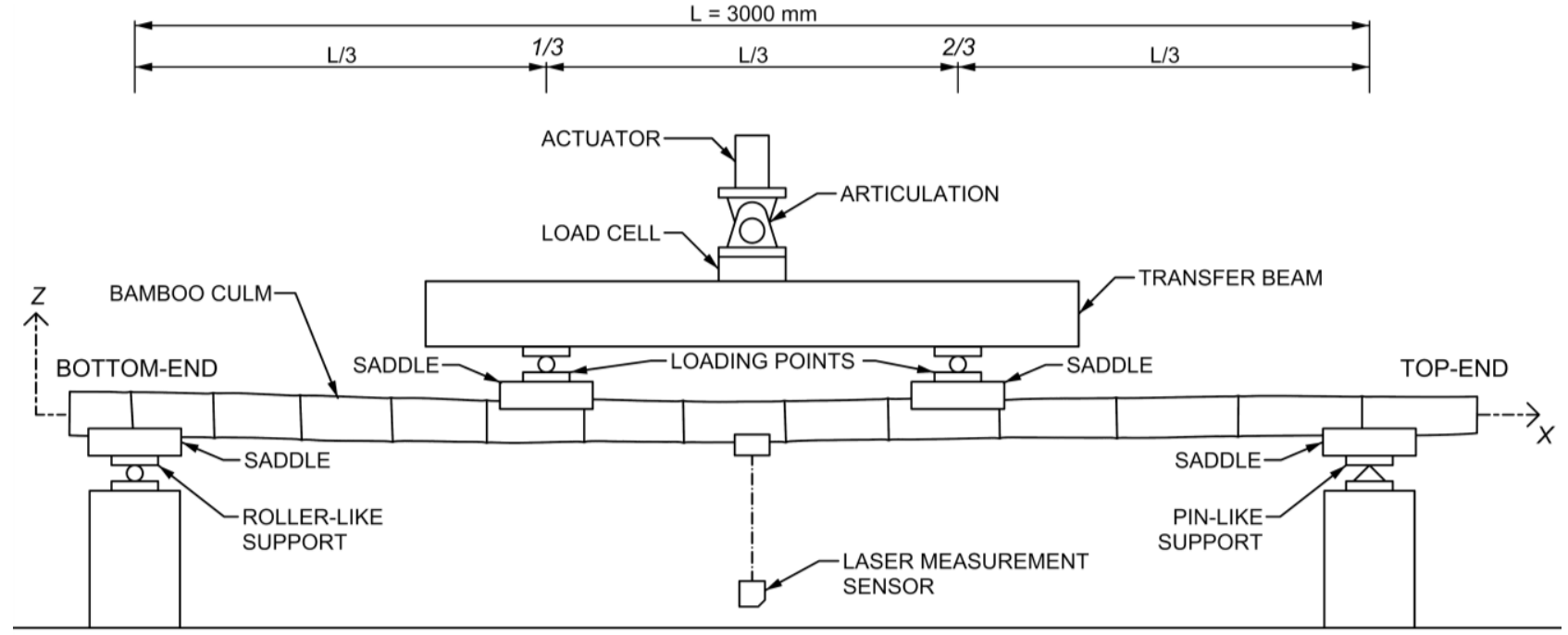

Figure 1. Four-point bending set-up

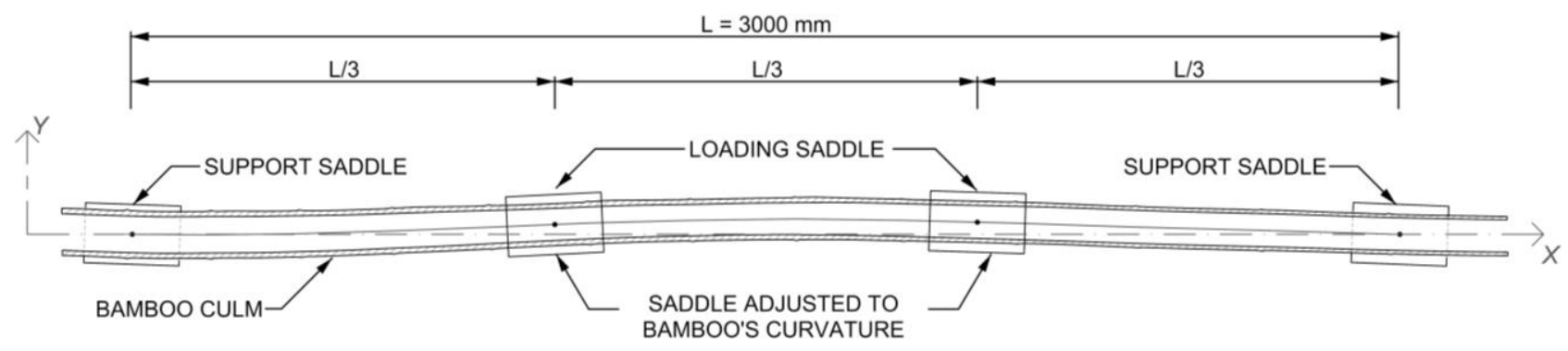

Figure 2. Top-view of bending set-up

\subsection{Experimental results}

The results of the experimental test, expressed in a load-deflection graph, are shown in Figure 3. Bamboo is characterised by its considerably high material elasticity, compared to other construction materials, such as wood or aluminium. This property was observed throughout the experimental test where the ultimate average displacement nearly reached $10 \%$ of the free span $(\mathrm{L} / 10)$. In addition, it was observed that there is an asymmetric deformation that might be related with the reduction of cross section towards the top end of the culm. This effect was allowed in the experiment due to an articulation between the actuator and the transfer beam. The load was equally distributed on the transfer beam, however the variation in section properties produced differential deformations at the loading points, thus a pronounced inclination in the transfer beam occurred (Figure 4). If the transfer beam was to be fixed to the actuator, the deformation might have been symmetric, but the load distribution between the two points of application would have created an asymmetric distribution of shear forces and bending moments.

In terms of failure, the five samples presented four cracks which developed suddenly along the central third of the culm, each crack distributed at approximately 90 degrees (Figure 5) around the pole's circumference. A high percentage of failure cases has been reported in the literature $[10,18]$ as caused by shear or crushing around the points of application of the loads. This failure behaviour does not represent the characteristic failure of a bending test of thin-walled tubes and might be caused by a local effect. 
The mechanical properties calculated from the test results were modulus of elasticity (E) and ultimate strength $\left(\sigma_{u}\right)$ based on the equations and recommendations in the standard [13]. It is important to note that these mechanical properties are only equivalent, average properties due to the longitudinal variation of the pole's fibre content along their length [6]. The codified calculation procedure for $\mathrm{E}$ is ambiguous on the definition of the linear portion of the load-deflection diagram. According to this standard [13], E is "given by the slope of a linear part of the diagram", however, the definition of that linear part is open to interpretation.

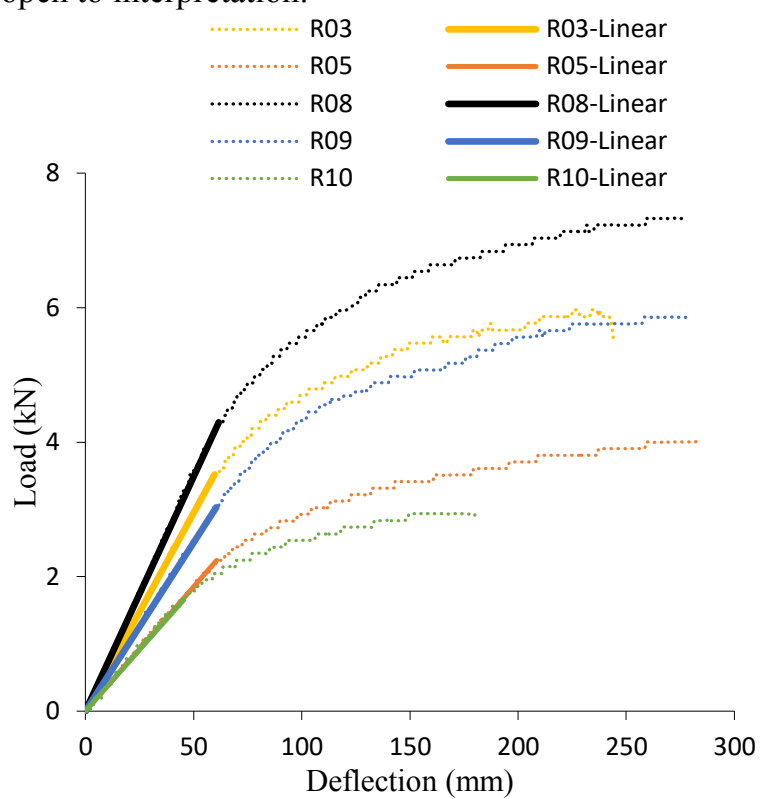

Figure 3. Load-deflection diagram for bending test with linear regression adjusted to $\mathrm{R}^{2}=0.99$

In this study the linear part of the load-deflection diagram was defined through a simple linear regression analysis of the load-deflection data (Figure 3), whereby a minimum coefficient of determination $\left(\mathrm{R}^{2}\right)$ of 0.99 was adopted as an objective cut-off threshold to define the linear portion of the diagram.

The modulus of elasticity (E) calculated for each pole, together with the ultimate strength $\left(\sigma_{u}\right)$ are shown in Table 2. The wide range of results is noticeable considering that the properties were obtained from a small quantity of samples, which comprised similar physical characteristics, such as specie, region, age, moisture content and average diameter. The difference between the minimum and maximum magnitude of $\mathrm{E}$ and $\sigma_{\mathrm{u}}$ was about $22 \%$, and $44 \%$ respectively. These results partly explain the large variability of mechanical properties found in the literature and raise some questions on the conservatism required to define a single characteristic value on a highly variable natural material. According to the literature $[5,6]$, this variability has been linked to the internal proportion of the constituent materials of bamboo (e.g. fibre and matrix) that tend to vary significantly not only between culms of the same species, but also along and across a single culm (functionally graded material). An alternative approach to characterise the material properties of bamboo could consider the individual variability of the constituent elements of bamboo's anatomic microstructure and relate that variability to the macroscopic behaviour.

Table 2. Material properties

\begin{tabular}{ccc}
$\begin{array}{c}\text { Moso } \\
\text { Bamboo }\end{array}$ & $\begin{array}{c}\mathrm{E} \\
\mathrm{MPa}\end{array}$ & $\begin{array}{c}\sigma \mathrm{u} \\
\mathrm{MPa}\end{array}$ \\
\hline R03 & 10851 & 55.7 \\
R05 & 10916 & 54.1 \\
R08 & 13246 & 70.3 \\
R09 & 13900 & 74.4 \\
R10 & 11056 & 41.2
\end{tabular}

Moreover, the deviations from the standard bending test caused by the poles' inherent geometric variability are very likely to affect and increase the uncertainty of the test results if this variability is neglected and poles are idealised as straight, prismatic tubes. The variation in the shape of bamboo poles has been measured and described by several authors $[6-8,12]$, but the complexity of their geometry is a limitation for conventional measuring methods. Section 3 described the methodology and results of a series of finite element analyses based on the accurate digital models of the five bamboo samples. The analyses were performed in order to identify the structural effects on bending behaviour produced by bamboo's natural variation in cross-section properties and out-ofstraightness. 


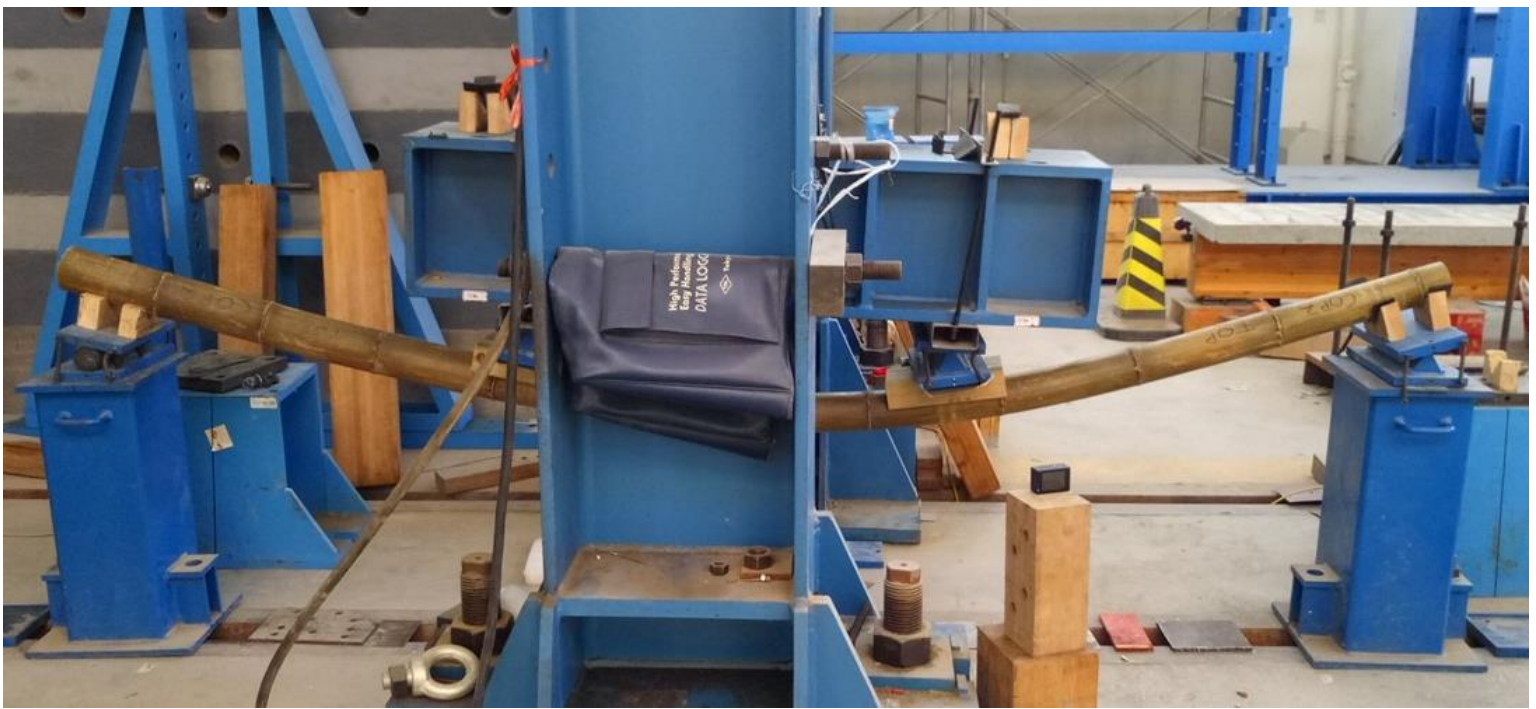

Figure 4. Asymmetric deformation towards top side of the culm

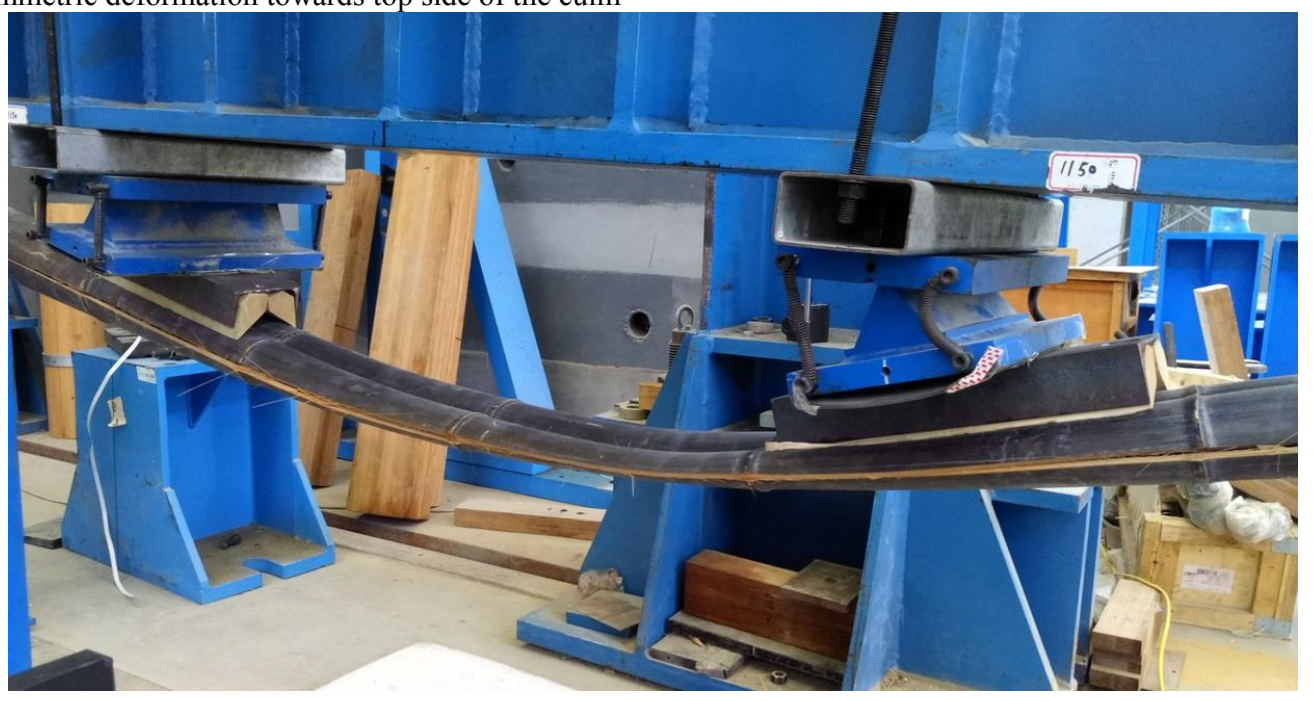

Figure 5. Typical failure of bamboo poles during bending test

\section{Finite element analyses}

\subsection{Digitisation and finite element modelling of bamboo poles}

Prior to the mechanical test, each bamboo was digitised using a mid-range Artec Eva 3D scanner [19] which is a hand-held device that operates based on structured light sensor technology [20]. The scanner was operated using a laptop Dell XPS 15 equipped with an Intel i76700HQ CPU @ $2.66 \mathrm{GHz}, 16 \mathrm{~GB}$ of installed memory and a dedicated video card Nvidia GTX GeForce $960 \mathrm{~m}$ with 4 GB of memory, whilst postprocessing of point cloud into a polygon mesh was carried out using Artec's proprietary software Artec Studio 12 [19] in a work station Dell Precision with an Intel Xeon E5-1620v3 CPU@3.5 GHz, 32 GB of memory and a dedicated video card Nvidia Quadro K2200 with 4 GB of memory. The set-up and configuration of the equipment were arranged in a way that allowed the bamboo to be scanned by simultaneous rotational and translational movement, therefore, the acquired point cloud followed an overlapped helicoidal pattern. Once the acquisition process was finished, the post-processing of the 3D data point took place, which generated an accurate digital representation of bamboo's surface (Figure 6). The digital mesh model was comprised of an external surface that covered the entire bamboo geometry and a portion of the internal surface at each end. According to the literature $[6-8,18]$ the wall-thickness tends to decrease linearly from bottom to top, therefore, the captured portion of the internal section was used to estimate the wall-thickness along the length of the pole by linear interpolation.

A polygon mesh model of a bamboo pole can offer advantages for design, visual grading and manufacturing processes but it would not be suitable for a FE analysis of a basic skeletal structural model. The polygon mesh was therefore processed into a line model suitable for structural analysis. This discretisation consisted in defining the centroidal axes of the poles joining the centroids of each nodal section. 
Sections of pole between nodes (internodes) represent single FE elements whose properties were determined based on those of the cross-sectional properties extracted at the mid-point of each internode (Figure 7). This simple line-based structural model is suitable to capture the geometric variation of diameter, thickness, area, inertia and out-of-straightness along the culm.

The discretisation and calculation of the geometric parameters for the structural model of all bamboo poles were performed using the software Grasshopper [21] and GeometryGym [22], both plug-ins for the computer aided design (CAD) software Rhinoceros 3D [23]. The FE modelling data was exported to the structural analysis software GSA [24] which was used to carry out the parametric study on the effect of the poles' geometric variability on their behaviour under four-point bending. This parametric study included four different models.

Figure 6. Polygon mesh and texture model of a Moso bamboo

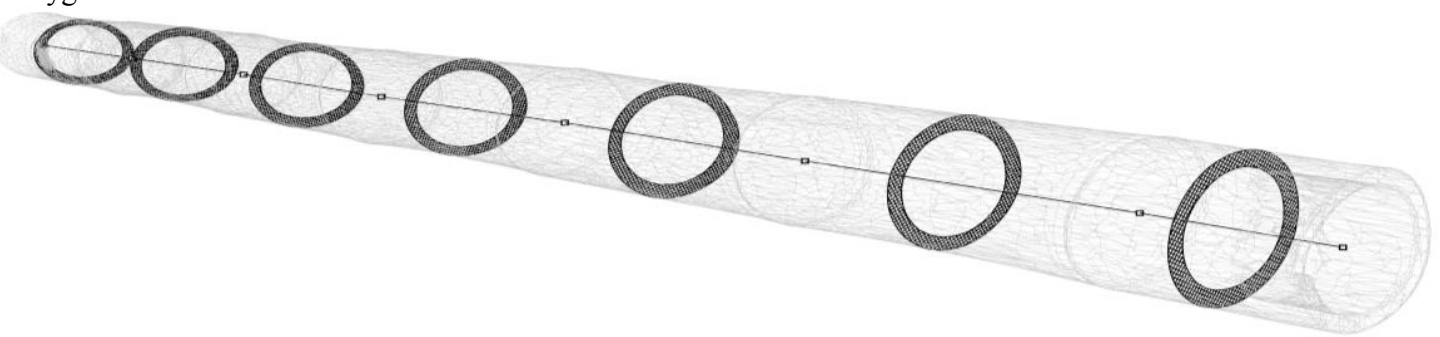

Figure 7. Cross-section curves at every internode and centre-line axis extracted from the mesh model

Model $A$ was developed based on single average geometric properties assuming the bamboo poles to be idealised straight and prismatic tubes (Figure 8A). This model was built based on the general recommendations given in [13] for the determination of geometric properties, and therefore was used as the benchmark for this study. Model $B$ was based on average but discretised geometric properties at each internode (varying from bottom to top) and, as in Model $A$, assuming the poles to be straight and prismatic (Figure $8 \mathrm{~B})$. Model $B$ was used to analyse the structural effects of the natural taper in bamboo poles. Model $C$ follows the same discretisation of Model $B$ but the section properties are not those of an equivalent circular tube but those calculated from the actual cross-sections of each internode (Figure 8C). As a result, the magnitude and direction of the principal moments of inertia $\left(\mathrm{I}_{1}, \mathrm{I}_{2}\right)$ of these sections vary along the length of the pole (Figure 9). Finally, Model $D$ incorporates the actual geometry of the pole centroidal axis idealised through straight elements at the internodes and changes of direction at every node. As such, Model $D$ includes discrete elements with irregular cross-sections varying along the culm at every internode considering the natural deviation of the poles centroidal axis from a straight line (i.e. out-of-straightness) (Figure 8D).

The calculation of the section properties for idealised or irregular cross-section for each model was performed according to the equations in Table 3. The material properties for each model were assigned according to the results obtained from the bending tests performed on the physical poles (R03-R10) as shown in Table 2. The material for these five samples was treated as isotropic and a static linear-elastic analysis was performed. The configuration of the supports was the same throughout the four models for each bamboo sample. The support system consisted in a simple supported beam (roller-pin arrangement shown in Figure 8) without any rotational restraint about the global $\mathrm{Y}$ and $\mathrm{Z}$ axes to capture the three-dimensional behaviour of the poles caused by spatial variation of their geometry. However, due to this variation, torsional restraints at both ends of the beam were included in the analysis to model the friction developed in the actual supports of the experimental set up. This friction prevented the poles from collapsing as the applied loads lay outside the vertical plane passing through both supports (Figure 8D). 


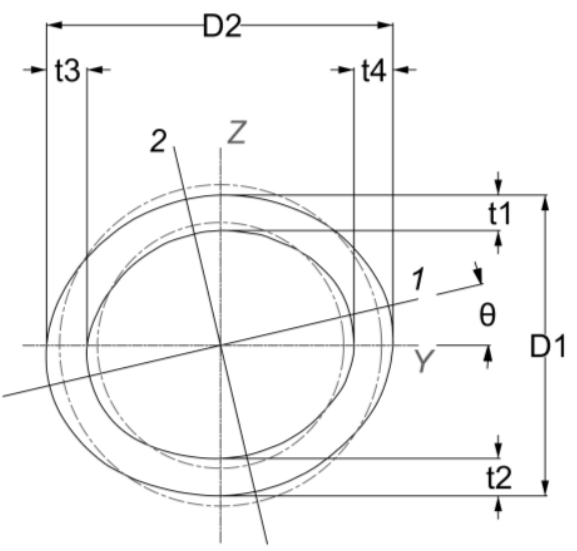

Figure 9. Cross section of actual pole cross section and equivalent average circular tube (dotted line)

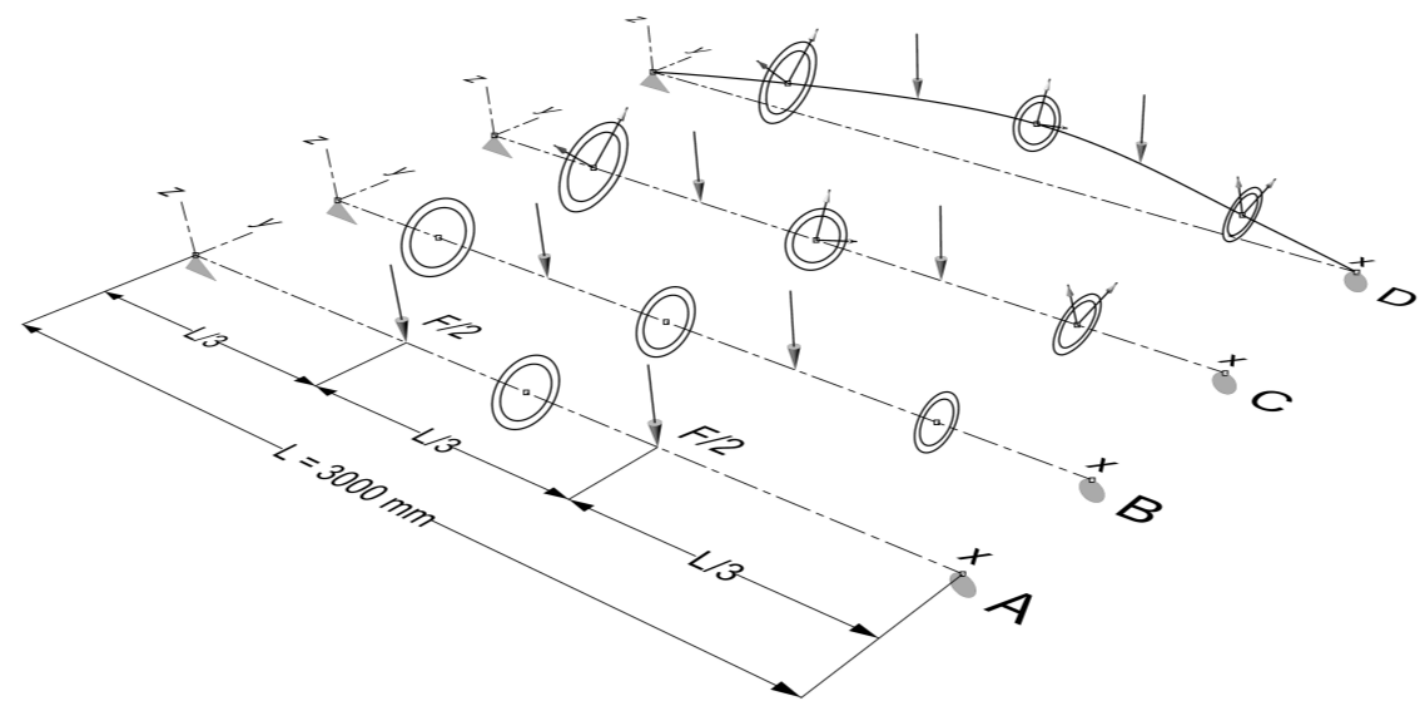

Figure 8. Diagram of the parametric structural FE models (A, B, C, D). (Not to scale)

Table 3. Equations to calculate section properties

\begin{tabular}{|c|c|c|}
\hline Nomenclature & Description & Formula \\
\hline $\mathbf{A}_{\mathbf{e}}$ & External cross-section area & \\
\hline $\mathbf{A}_{\mathbf{i}}$ & Internal cross-section area & \\
\hline $\mathbf{I} \mathbf{x}_{\mathbf{m}}$ & Second moment of area $\mathrm{X}$ axis & Calculated directly from CAD software using \\
\hline $\mathbf{I y} \mathbf{y}_{\mathbf{m}}$ & Second moment of area $\mathrm{Y}$ axis & polygon mesh \\
\hline $\mathbf{I} \mathbf{z}_{\mathbf{m}}$ & Second moment of area $Z$ axis & \\
\hline $\mathbf{I y z} \mathbf{z}_{\mathrm{m}}$ & Product moment of area & \\
\hline D & Idealised equivalent diameter & $D=\sqrt{\frac{4 A_{e}}{\pi}}$ \\
\hline Di & Idealised equivalent internal diameter & $D_{i}=\sqrt{\frac{4 A_{i}}{\pi}}$ \\
\hline $\mathbf{T}$ & Idealised equivalent thickness & $T=\frac{D-D_{i}}{2}$ \\
\hline $\mathbf{A}$ & Idealised area & $A=\frac{\pi}{4}\left(D^{2}-(D-2 T)^{2}\right.$ \\
\hline $\mathbf{I y}, \mathbf{I z}=\mathbf{I}$ & Idealised second moment of area & $I=\frac{\pi}{64}\left(D^{4}-(D-2 T)^{4}\right.$ \\
\hline $\mathbf{J}$ & Idealised polar moment of area & $J=\frac{\pi}{32}\left(D^{4}-(D-2 T)^{4}\right.$ \\
\hline $\mathbf{A}_{\mathbf{r}}$ & Irregular area & $A_{r}=A_{e}-A_{i}$ \\
\hline $\mathbf{I}_{1}, \mathbf{I}_{2}$ & Principal second moment of area & $I_{1}, I_{2}=\frac{1}{2}\left[I_{y m}+I_{z m} \pm \sqrt{\left(I_{y m}-I_{z m}\right)^{2}+4 I_{y z m}^{2}}\right]$ \\
\hline$\theta$ & Angle from $\mathrm{Y}$ axis to principal mayor axis & $\theta=\frac{1}{2} \tan ^{-1}\left(\frac{2 I_{y z m}}{I_{z m}-I_{y m}}\right)$ \\
\hline
\end{tabular}




\subsection{Geometric variation}

The geometric variability of all poles was automatically calculated from the scanned model. Based on the growth features of bamboo poles, the discretisation of the acquired digital models consisted of linear segments within the internodes with changes of direction only at the nodes. The cross-section properties of each discrete element were extracted at the middle of each internode and stored in a database. This database can be adopted as a very powerful tool for any subsequent geometric modelling, structural analysis or optimisation processes. Figure 10 shows the variation of diameter along the normalised length of the poles. For each sample, the average idealisation proposed by [13] is plotted as an average single point (diamond-point) at the poles' mid-point. Evidently, the average value of the diameter does not provide any information on the real variation of individual poles. Also, it is clear that not even a linear regression would accurately capture the variation in diameter along their length.

One of the most complex parameters to measure using conventional tools is the out-of-straightness, however, using the text database containing the centroid coordinates of each cross-section, the calculation of the maximum out of straightness was a straightforward process. The maximum out-ofstraightness was found by simply defining a reference straight line from the first to the last node of the pole. The maximum perpendicular distance between any centroidal node and this line defines the maximum outof-straightness of the pole. A set of orthogonal axes is defined as shown in Figure 11.

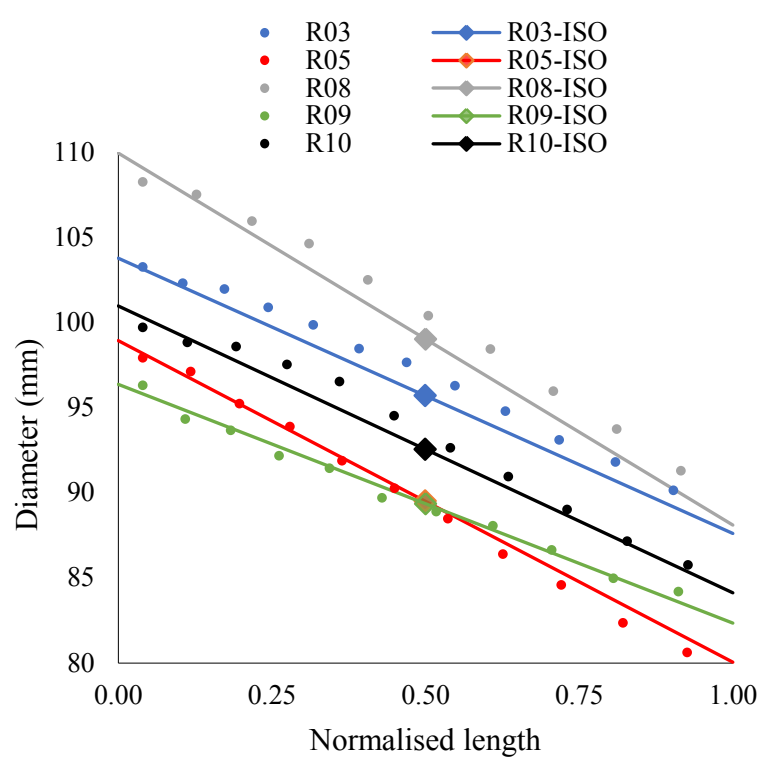

Figure 10. Variation of diameter along the length of bamboo, compared to the average single-value proposed by ISO22157-1 [13]

The direction of axis Xo coincides with that of the reference line between the pole end nodes. Axis $Z o$ is perpendicular to $X o$ and passes through the node which defines the pole maximum out-of-straightness, whereas the direction of $Y o$ follows the right-hand rule. These set of axes are used to demonstrate the natural variation of the position of the centroidal axis of each pole projecting the coordinates of the centroidal axes onto the XoYo and XoZo planes as shown in Figures 12 and 13. The maximum out-of-straightness for each bamboo sample is plotted as a single point in each plane, noting that all poles present a pronounced double curvature which can have a significant effect on their structural behaviour and represents a further challenge for manual measurements. The location at which the outof-straightness is maximum only coincides with the pole mid-point for sample R10 in the XoZo plane. The range of out-of-straightness in the XoZo plane varies from -7.0 to $-37.2 \mathrm{~mm}$ and approximately 10 to -10 $\mathrm{mm}$ in plane $\mathrm{XoYo}$.

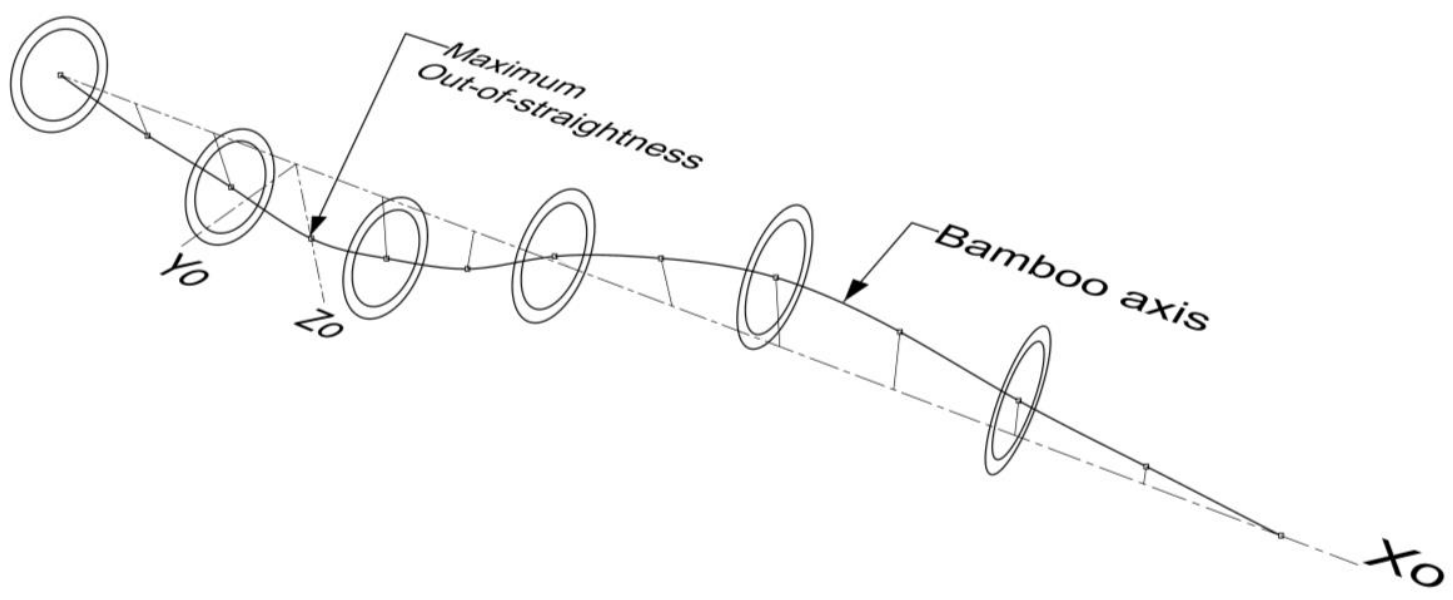

Figure 11. Diagram showing process to define the maximum out of straightness and local Xo, Yo \& Zo directions. (Yo \& Zo directions are ten times scaled for clarity) 


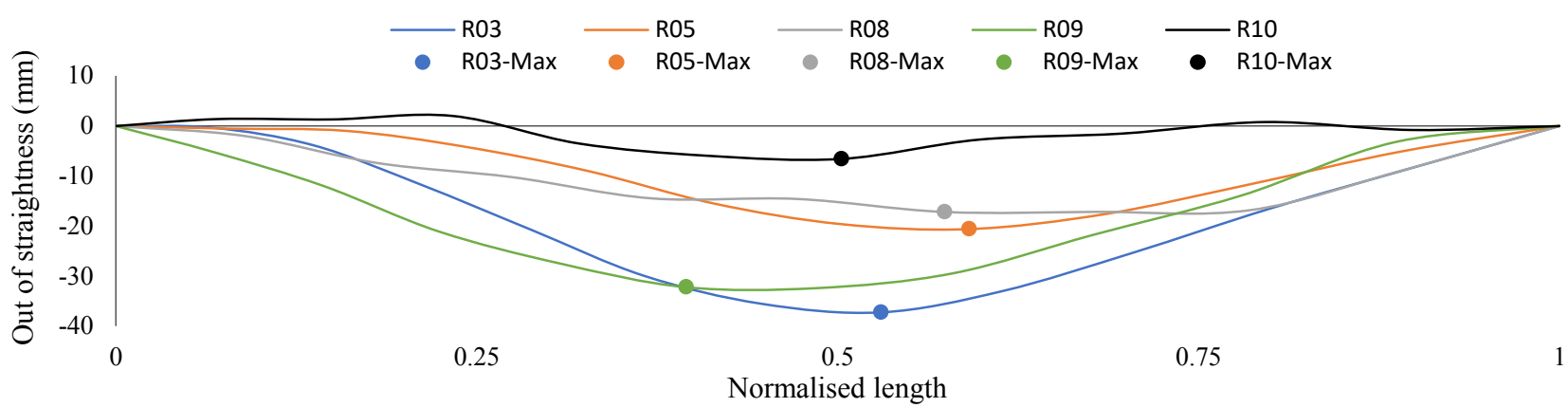

Figure 12. Out-of-straightness in the XoZo plane and maximum absolute deflection per sample

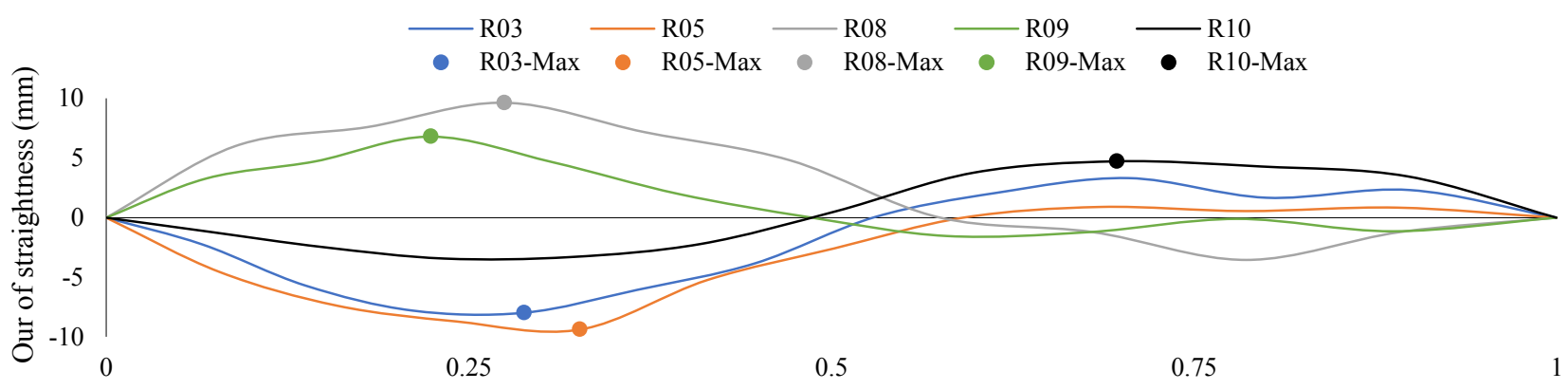

Normalised length

Figure 13. Out-of-straightness in the XoYo plane and maximum absolute deflection per sample

The cross-section properties of a bamboo pole can be calculated as those of an equivalent circular tube idealisation using conventional measuring tools to measure discrete diameters and thicknesses [13] (Table $3)$. In contrast, the cross-section properties extracted from the digitised mesh model represent the properties of the actual cross section of the poles accurately calculated through CAD software. As shown in Figure 10 , the principal moments of inertia $\left(\mathrm{I}_{1}, \mathrm{I}_{2}\right)$ of the actual cross sections will have different magnitudes and orientation along the pole compared with an idealisation of a circular tube. Figure 14 shows the average idealised moment of inertia (I) for each of the samples, compared to the actual major and minor moment of inertia $\left(\mathrm{I}_{1}, \mathrm{I}_{2}\right)$ for each sample. It was found that when considering the properties of the irregular cross-sections there was a significant variation of up to $16 \%$ compared to the idealised values. Figure 15 shows the variation of the orientation angle $(\theta)$ of the principal axis for the crosssections on each individual sample. The variation in angle range for the samples was significant, and in all cases the range exceeded 35 degrees. The results of all cross-section properties occurred in a random manner along the length of the five samples, so that specific pattern or trend could not be associated with the specific geometric variability.

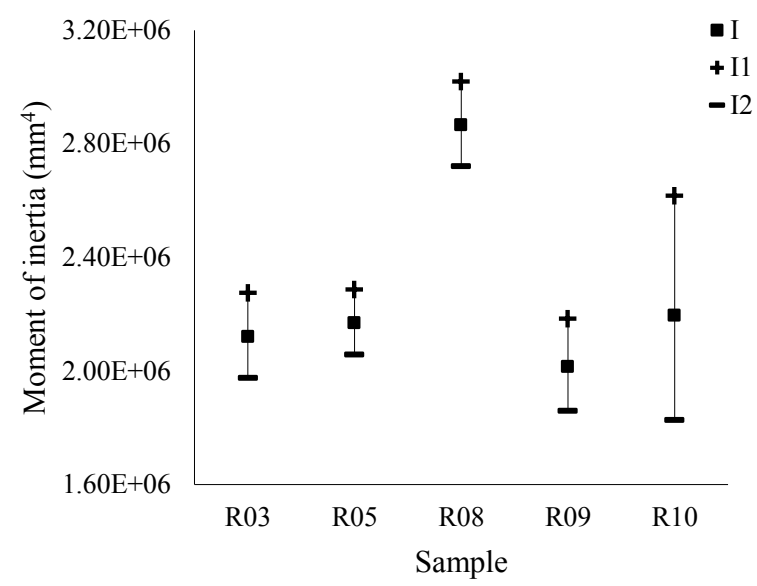

Figure 14. Average moment of inertia comparison between idealised (I) and real cross-section $\left(I_{1} \& I_{2}\right)$

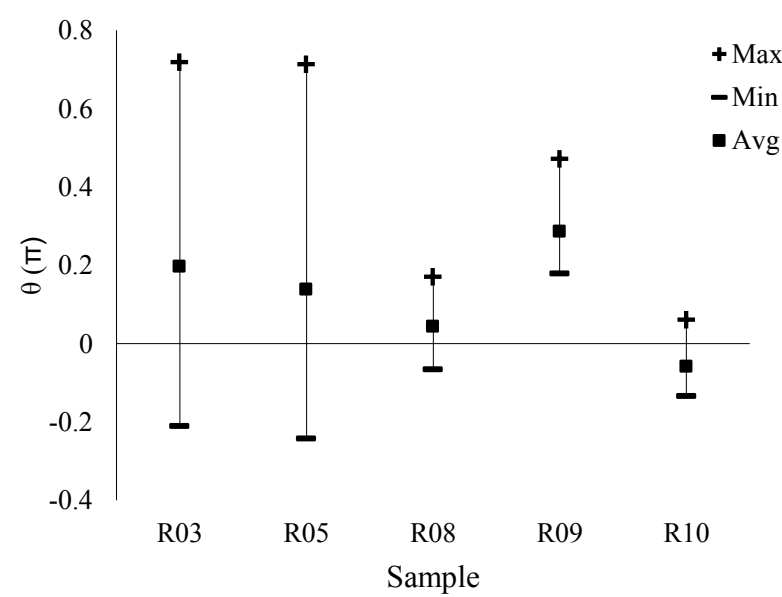

Figure 15. Variation range of the principal axis orientation angle $(\theta)$ per sample 


\subsection{Structural effects}

The geometric parameters extracted from the digitised model described in Section 3.2 were systematically implemented into structural models of five bamboo culms (Models $A, B, C$ and $D$ ). A structural analysis was performed for each model and the different effects were recorded and compared. The global orthogonal system adopted for the structural analysis was that shown in Figure 9, where $X$ axis was aligned with the supports and $Y \& Z$ formed a plane perpendicular to $X$. A normalised force $(F)$ was applied to all the models in $-Z$ direction (Figure 8). According to Figure 9, the principal local axes for the cross-sections in models $C \&$ $D$ were denominated $1 \& 2$ for the major and minor axis respectively.

The first parameter to analyse was the deflection in the $\mathrm{Z}$ direction for Models $B, C \& D$ compared to the maximum displacement of the idealised Model $A$, normalised to 1.0. Figure 16 shows that the maximum displacement for models $B \& C / D$ had a considerable difference with model $A$, ranging from 0.5 to $14 \%$, which in average represented an increment in displacement of $5.7 \%$. The variation in the geometry of the bamboo not only affected the magnitude of the maximum deflection, but also the position at which it occurs. Compared to Model $A$, the maximum displacement of models $B, C \& D$ was shifted by approximately $75 \mathrm{~mm}$ towards the top end of the pole due to reduction in the cross-section. A secondary effect produced by the reduction in cross-section properties was noticed by a differential deflection obtained in the loading points (at $1 / 3$ and $2 / 3$ of the span). This effect is shown in Figure 17, where it can be observed that the deflection at $2 / 3$ of the span (towards the top-end of the pole) is always bigger than that obtained at $1 / 3$ (towards the bottom-end). Consequently, the transfer beam would have an inclination (Figure 4) towards the top end of the pole with an average slope of $7 \%$ for the five samples. This is an important effect that should be taken into account for the correct design of an experimental test, as longitudinal components of the applied forces are likely to be generated along the culm.

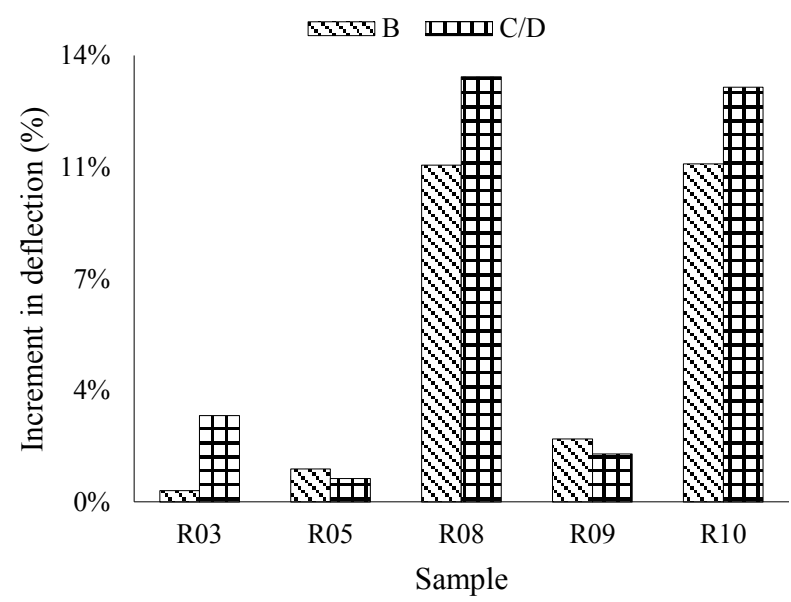

Figure 16. Increment (\%) of the maximum deflection in $Z$ direction for models $B \& C / D$ compared to model $A$

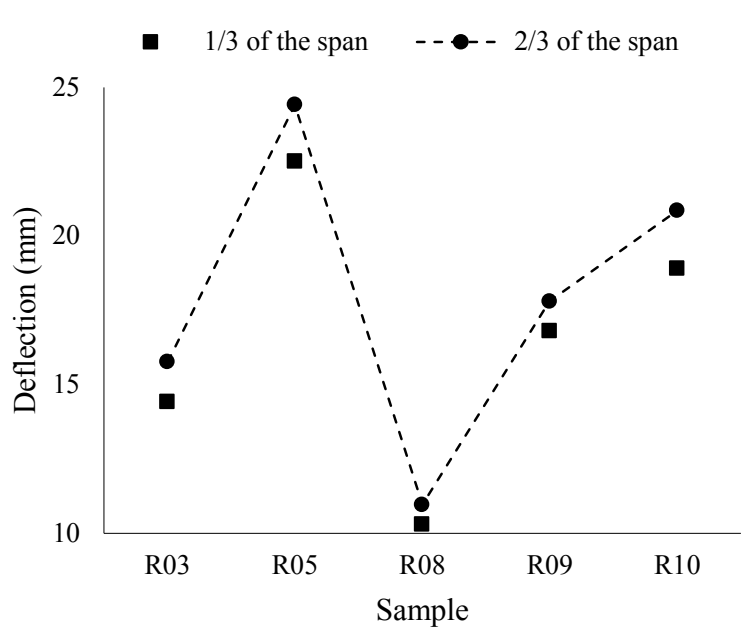

Figure 17. Differential deflection in loading points located at $1 / 3$ (bottom-end) and 2/3 (top-end) of the span

These numerical findings agreed with the physical observations of asymmetric deformation during the bending test and highlighted the complexity of measuring the behaviour from physical tests, as the position of the maximum deflection does not necessarily occur at mid-span. Also, it is important to consider that adopting the mid-span deflection for the calculation of the equivalent $\mathrm{E}$ of the pole [13], might lead to unconservative results.

For the idealised prismatic model, the deflection in the $X \& Y$ directions was zero for both, whereas the models that included the irregularity of the bamboo presented values that differed from zero. These values were not considered significant in any of the five samples subjected to four-point bending, however, due to the considerable geometric variation found in bamboo poles, this out-of-plane behaviour should be quantified and assessed.

As expected, the idealised prismatic tubes (Models $A$ $\& B$ ) only presented shear forces in the global $Z$ direction (considered minor axis) and bending moment about the global $Y$ axis (considered major axis). However, for Models $C \& D$ the forces were decomposed into local, major and minor directions, according to the orientation angle $(\theta)$ of the principal local axis for each individual cross-section. Figure 18 shows how shear forces (V) and bending moments (M) of Models $C \& D$ are decomposed into local major and minor axes, compared to the normalised value (1.0) for $\mathrm{V}$ and $\mathrm{M}$ of Model $A$. Without exception, all the samples showed that the cross-section is subjected to biaxial effects due to the irregularity of the cross-section shape. This produces shear forces and bending moments around two principal axes of inertia, creating complex state of stresses that cannot be quantified without the aid of computer software. Moreover, in the case of bending moment for samples R05, R08 and R09, the largest component of $\mathrm{M}$ is applied around the minor axis of the cross-section which will therefore create higher stresses when compared to idealised cross-sections. 


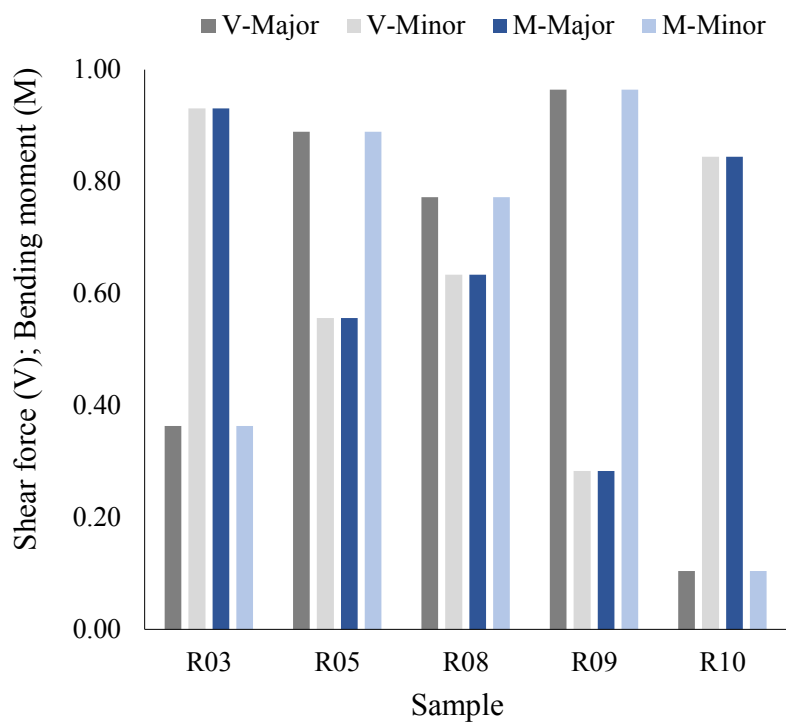

Figure 18. Shear forces (V) and bending moment (M) components (major and minor) of models $C \& D$ in comparison with normalised value (1.0) for $\mathrm{V}$ and $\mathrm{M}$ of Model $A$.

For Model $D$, torsional effects were developed due to the implementation of the natural geometry of the pole which lies outside the vertical plane containing both supports (Figure 8D). However, according to the physical tests and the analytical results, these torsional effects are not significant for the samples in this study as no physical rotation at the supports was recorded during tests which suggests that friction developed at the supports was enough to prevent rotation. In spite of the non-significant torsional effects for this study, the quantification and assessment of torsion should be performed due to the random nature of the geometric variation found in bamboo poles.

\section{Conclusion}

The focus of this work was to identify and analyse the structural effects caused by the natural geometric variation found in bamboo poles. Five Moso bamboo poles were subjected to a four-point bending test to identify and quantify the effect of these geometric variations on their overall behaviour. Prior to testing, the bamboo poles were 3D scanned to generate digital polygon mesh models of the five samples capable of describing their geometry to a high degree of accuracy. These models were post-processed to extract the key geometric parameters required to create a skeletal model of each pole and conduct a finite element analysis.

The results showed that the basic geometric parameters, such as diameter, thickness, area and moment of inertia differ considerably from average values obtained by the idealisation of bamboo poles as straight circular tubes. It was also found that the inherent geometric variation of the poles had a significant effect on their structural behaviour with differences in maximum vertical displacements of up to $14 \%$. In addition, calculation of the modulus of elasticity of the poles based on the mid-span displacement, as per the current standard recommendations [13], could lead to unconservative differences, compared to the value calculated based on the maximum beam displacement not occurring at the pole mid-span due to its natural taper and change in cross section properties. Furthermore, the geometric variability of bamboo poles can lead to significant asymmetric deformations and distribution of internal forces and moments compared to the theoretical behaviour of an idealised circular, straight and prismatic tube assumed in current standards [13]. There is a significant difference in the cross-sectional properties between this tubular idealisation and those based on the actual pole geometry. In particular, the principal second moments of inertia of the actual cross sections are significantly different to the single value calculated based on a tubular idealisation. Also, the orientation of these principal axes continually changes along the pole according to its natural dimensional variation.

This study shows the significance of taking into account the actual spatial geometry of bamboo poles for the development of experimental tests and their accurate structural analysis. The proposed digital technologies and methods to efficiently and accurately capture and process the geometric variability of bamboo poles can help us improve our understanding of this natural structural element. On the whole, the principles on which this work is based can be further applied to develop new digital workflows to support the design, modelling, analysis and fabrication of bamboo structures.

\section{Acknowledgements}

The work presented in this paper was supported by: the UK Engineering and Physical Sciences Research Council (EPSRC) (Grant Nos: EP/M017702/1 \& EP/P510890/1); the China-UK-China Joint Research and Innovation Partnership Fund $\mathrm{PhD}$ Placement Program (Grant No: UK-276080305). The authors would like to thank Nanjing Forestry University for the support provided on the performance of the experiments. They also would like to thank the generosity of GeometryGym Ltd for providing an educational license of their computer software.

\section{References}

1. UN-Habitat projects, UN-Habitat 53. United Nations Human Settlements Programme. Nairobi, Kenya (2013)

2. World urbanization prospects, the 2014 revision: highlights. New York: United Nations, 2014

3. W Liese. Research on bamboo. Wood Science and Technology 21: 189-209 (1987). https://doi.org/10.1007/BF00351391

4. D Grosser and W Liese. On the anatomy of Asian bamboos, with special reference to their vascular bundles. Wood Science and Technology 5: 290-312 (1971). https://doi.org/10.1007/bf00365061 
5. PG Dixon and LJ Gibson. The structure and mechanics of Moso bamboo material. Journal of the Royal Society Interface 11: 20140321 (2014)

6. S Amada, Y Ichikawa, T Munekata, Y Nagase and $\mathrm{H}$ Shimizu. Fiber texture and mechanical graded structure of bamboo. Composites Part B 28B: 13-20 (1997). https://doi.org/10.1016/b978-044482548$\underline{3 / 50119-0}$

7. KF Chung and WK Yu. Mechanical properties of structural bamboo for bamboo scaffoldings. Engineering Structures 24(4): 429-442 (2002). https://doi.org/10.1016/s0141-0296(01)00110-9

8. K Ghavami and LE Moreira. The influence of initial imperfection on the buckling of bamboo columns. Asian journal of Civil Engineering (Building and Housing) 3(3 \& 4): 1-16 (2002)

9. B Sharma, KA Harries and K Ghavami. Methods of determining transverse mechanical properties of full-culm bamboo. Construction and Building Materials 38: 627-637 (2013)

10. D Trujillo, S Jangra and JM Gibson. Flexural properties as a basis for bamboo strength grading. Proceedings of the Institution of Civil Engineers, Structure and Buildings 170(SB4): 284-294 (2017). https://doi.org/10.1680/jstbu.16.00084

11. WK Yu, KF Chung and SL Chan. Column buckling of structural bamboo. Engineering Structures 25: 755-768 (2003)

12. JJA Janssen. Designing and building with bamboo. International Network for Bamboo and Rattan, Beijing China (2000)

13. ISO (International Organization for Standardization). ISO 22157-1:2004: Bamboo - Determination of physical and mechanical properties - part 1: requirements. ISO, Geneva, Switzerland (2004)

14. TY Lo, HZ Cui, PWC Tang and HC Leung. Strength analysis of bamboo by microscopic investigation of bamboo fibre. Construction and Building Materials 22: 1532-1535 (2008)
15. R Lorenzo, C Lee, JG Oliva-Salinas and MJ Ontiveros-Hernandez. BIM Bamboo: a digital design framework for bamboo culms. Proceedings of the Institution of Civil Engineers, Structure and Buildings 170(SB4): 295-302 (2017). https://doi.org/10.1680/jstbu.16.00091

16. Wikipedia. Polygon mesh. (2018) See https://en.wikipedia.org/wiki/Polygon mesh (Accessed 15/10/18)

17. D Taylor, B Kinane, C Sweeney, D Sweetnam, P O'Reilly and K Duan. The biomechanics of bamboo: investigating the role of the nodes. Wood Science and Technology 49: 345-357 (2015). https://doi.org/10.1007/s00226-014-0694-4

18. TS Paraskeva, G Grigoropoulos and EG Dimitrakopoulos.Design and experimental verification of easily constructible bamboo footbridges for rural areas. Engineering Structures 143: 540-548 (2017). https://doi.org/10.1016/j.engstruct.2017.04.044

19. Artec 3D (2018) See https://www.artec3d.com/ (accessed 15/10/2018).

20. D Fofi, T Sliwa and Y Voisin. A comparative survey on invisible structured light. Proc. SPIE Machine Vision Applications in Industrial Inspection XII 5303: 90 (2004). https://doi.org/10.1117/12.525369

21. Grasshopper 3D (2018) See https://www.grasshopper3d.com/ (accessed $15 / 10 / 2018)$

22. Geometry Gym Pty Ltd (2018) See https://geometrygym.wordpress.com/ (accessed $15 / 10 / 2018)$

23. RMNA (Robert McNeel and Associates) (2015) Rhinoceros Software Version 5.0. Robert McNeel \& Associates, Seattle, WA, USA

24. Oasys (2018) See https://www.oasyssoftware.com/products/structural/gsa-suite/ (accessed

$15 / 10 / 2018)$ 